\title{
Explotación Industrial de Pinnípedos en la Factoría de Bahía Tethis (Tierra del Fuego, Argentina): Una Aproximación Zooarqueológica
}

\author{
Industrial Exploitation of Pinnipeds in Tethis Bay Factory (Tierra del Fuego, Argentina): \\ A Zooarchaeological Approach
}

Martín Vázquez y Fernando Santiago ${ }^{\text {ii }}$

\section{RESUMEN}

Se presenta información zooarqueológica sobre una cama de huesos de pinnípedos, producto de las actividades de una factoría emplazada en Bahía Thetis (Tierra del Fuego), que explotó comercialmente las colonias emplazadas en la zona durante la década de 1940. Se describen los primeros trabajos de campo llevados a cabo en esta acumulación de huesos, cuyo objetivo fue obtener datos preliminares sobre su densidad, abundancia taxonómica y anatómica, así como efectuar ciertas observaciones tafonómicas tendientes a discutir aspectos vinculados a los procesos de formación de sitio. Si bien la composición taxonómicapuede considerarse casi mono específica y se interpreta que la gran mayoría de estos restos sonproducto de la faena en la factoría, los resultados inícialessugierenel carácter secundario de esta acumulación, la cual habría sido conformada por el trasporte ocasionado por las mareas de los restos descartados en la bahía.

Palabras clave: Pinnípedos, Explotación Industrial, Zooarqueología,Tierra del Fuego.

\section{ABSTRACT}

Zooarchaeological data about a sea lion bone bead from Thetis Bay (Tierra del Fuego) is presented herein. They are the result of the activities carried out by a factory that exploited commercially colonies settled in the area during the 1940s.First field work on this bone accumulation pointed to present preliminary data on density, anatomical and taxonomic abundance, as well as taphonomic observations with the goal to discuss aspects related to site formation processes. Though the taxonomic composition can be considered almost monospecific and is interpreted that the most of these remains resulted from the tasks in the factory, the former indicators suggest the secondary nature of this record, which would have been accumulated by tides, transport in the discarded remains.

Key words: Pinnipes, Industrial Exploitation, Zooarchaeology, Tierra Del Fuego.

\footnotetext{
${ }^{\text {i }}$ Museo del Fin del Mundo, Maipú 173. Ushuaia, Argentina. Correo-e:

vazquezmartin68@gmail.com

ii CADIC-CONICET, Bernardo Houssay 200. Ushuaia, Argentina.

Correo-e: ersant2@gmail.com
}

Recibido: 03-12-2012 - Revisado: 02-05-2013 - Aceptado: 27-02-2014 


\section{INTRODUCCIÓN}

Hacia fines del siglo XVIII comienza la caza industrial de lobos marinos en Sudamérica. En las Islas Malvinas se cazaron millones de lobos finos (Arctocephalus australis) por su piel, de tal manera que alrededor de 1800 eranprácticamente imposibles de localizar en estas regiones. Las campañas loberas eran una actividad tan lucrativa, que los barcosincursionaron hasta las islas Georgia y Shetland del sur (Stehberg y Cabeza 1987,Zarankin y Senatore 2007). Según los mencionados autores, el período de mayor actividad se registró entre 1819 y I83I y durante la segunda mitad del siglo XIX las cacerías se centraron en las ballenas. En la primera mitad del siglo $\mathrm{XX}$ el gobierno argentino reglamentó esta actividad otorgando concesiones para la explotación de mamíferos marinos, entre ellas para la factoría que se ubicó en Bahía Thetis (Tierra del Fuego), desde donde se explotaban alrededor de una docena de colonias de pinnípedos entre el río Luz y cabo San Diego' (Winograd 2008).

Bahía Thetis se ubica en el extremo este de la costa norte de Península Mitre, Tierra del Fuego, Argentina (Figura I). Durante prospecciones arqueológicas recientes, realizadas en el marco del Programa Arqueológico Costa Atlántica (PACA) (Vázquez et al. 2010) fueron registrados numerosos yacimientos arqueológicos en el área, entre ellos restos de naufragios históricos, restos de la Sub Prefectura que funcionó desde 1887 y los restos de la factoría que explotaba la grasa de pinnípedos en la década de 1940.

La grasa, muy utilizada para la fabricación de lubricantes pesados, tenía una importante demanda en el mercado de la época, durante la Segunda Guerra Mundial. Según información histórica, se llegaron a matar unos 30.000 animales en los años más productivos (Bruno Videla 1980; Winograd 1984). El faenamiento se realizaba principalmente en los lugares de caza, pero otros animales eran "arreados" y encerrados en corrales en las cercanías de la factoría, donde se los ultimaba, procesaba y se descartaban sus restos. Según Winograd (2008),la factoría trabajaba desde principios de primavera hasta principios de otoño, temporada en la cual vivían allí más de 200 personas. Mientras la productividad de la factoría crecía, la población de pinnípedos fue casi exterminada a fines de la década de 1940, hasta que en 1949 se prohibió la caza de lobos marinos en todo el territorio argentino. Según este autor, treinta y cinco años después del abandono del establecimiento no se observaban animales en la bahía. En la actualidad (fines del año 20II) hemos observado apostaderos de 0 . flavescensactivos en el área.

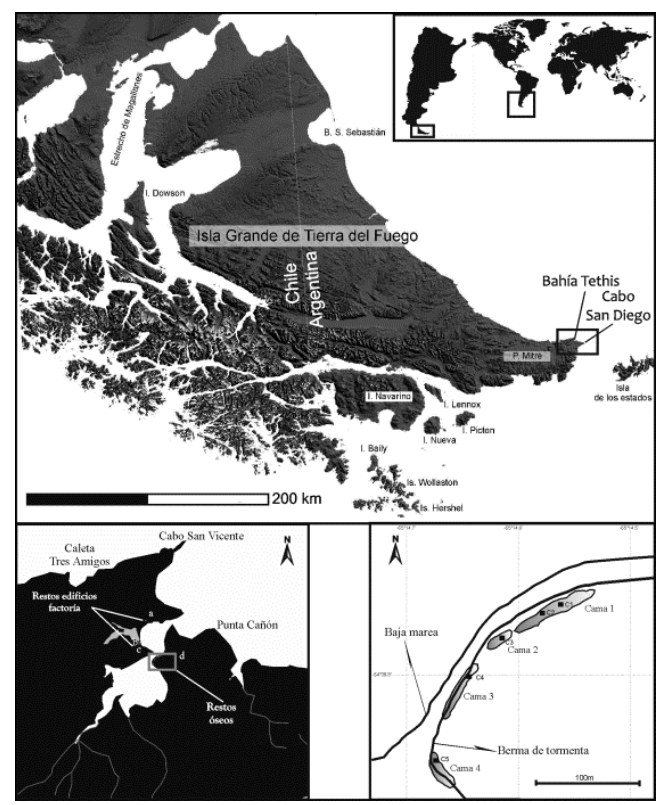

Figura 1: Ubicación de Bahía Tethis y el sitio analizado.

Figure 1: Bahia Tethis location of and the analyzed site.

El objetivo de este trabajo es presentar las observaciones realizadas sobre una gran acumulación de huesos producto de las actividades de la factoría, en las inmediaciones de las ruinas de los edificios históricos (Figura 2a,2by 2c). Se presentan datos sobre la composición taxonómica y anatómica, densidad de huesos por unidad de espacio y observaciones tafonómicas, con el objetivo de ofrecer una primera descripción de esta particular acumulación, así como discutir la acción de los distintos agentes y procesos implicados en su formación.

\section{MATERIALESY MÉTODOS}

El sitio está ubicado en la margen este del estrechamiento que divide la bahía Thetis exterior y el estuario interior, se trata de una gran acumulación 
de huesos de pinnípedos de unos $708 \mathrm{~m}^{2}$, también fue registrada una gran cantidad de huesos dispersos en superficie $o$ en proceso de enterramiento en las inmediaciones de los edificios históricos. Se delimitaron cuatro camas de huesos separadas por guijarros de playa, todas ellas depositadas detrás de la berma de tormenta actual (Figura I). Sus contornos fueron mapeados mediante GPS y las superficies de las acumulaciones fueron calculadas utilizando un sistema de información geográfica: Cama I. 460 $\mathrm{m}^{2}$, Cama $2.80 \mathrm{~m}^{2}$, Cama $3.150 \mathrm{~m}^{2}$ y Cama $4.18 \mathrm{~m}^{2}$. Por fuera de estas concentraciones relativamente discretas se observaron numerosos especímenes dispersos, incluso muy por encima de la línea de máximas mareas, así como diversos sectores donde se registraron huesos enterrados.

Es necesario destacar que, debido a la imposibilidad de trasladar los materiales y al escaso tiempo con que se contaba para trabajar en el lugar, se definió una estrategia sumamente expeditiva que nos permitió obtener una primera aproximación sobre este particular conjunto. Se plantearon 5 cuadrículas de muestreo de $|x|$ metro, dos de ellas en la denominada Cama I, la de mayor superficie y densidad, y una cuadrícula en cada una de las otras tres camas (Figura l y 2c). Aunque se constató la presencia de huesos enterrados en tres de las cinco cuadrículas, solamente se trabajó con el material de superficie. En estas unidades de muestreo se cuantificaron todos los especímenes y se realizaron identificaciones taxonómicas y anatómicas in situ, incluyendo criterios de lateralidad, tamaño y maduración.Para realizar las estimaciones taxonómicas y anatómicas se utilizaron las medidas convencionales en análisis zooarqueológicos NISP, MNI, MNE- (Lyman 1994, Mengoni Goñalons 1999). Desde el punto de vista tafonómico, en cada cuadrícula se seleccionaron al azar las tibias y cúbitos como elementos testigo, en los cuales se identificaron alteraciones por meteorización y modificaciones por carnívoros, roedores, raíces y fracturas en estado seco. Asimismo se buscaron huellas de corte, raspados, evidencia de fracturas, negativos de lascados y alteraciones por fuego (Fisher 1995, Lyman 1994, Mengoni 1999).

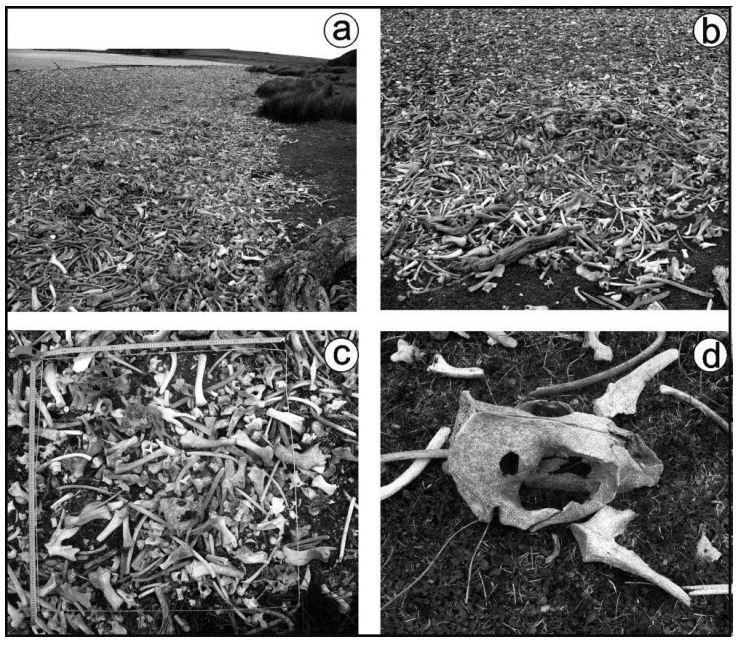

Figura 2: a-b) Diferentes vistas de la cama de huesos; c) detalle de una de las cuadriculas; e) cráneo con perforación.

Figure 2: $a-b)$ Different views of the bone bed: $c$ ) Detail of one of the grids; d) Sea lion skull with perforation.

\section{RESULTADOS}

En las 5 cuadrículas se identificó un total de 2141 especímenes, de los cuales el $82,8 \%(N=1772)$ corresponden a pinnípedos, I,7\% $(\mathrm{N}=36)$ a aves, $0,4 \%$ a Lama guanicoe $(\mathrm{N}=8)$ y un espécimen a Bos taurus, restando un $15,1 \%(N=324)$ que no fue posible asignar a ninguna categoría taxonómica más excluyente que vertebrados.

En la tabla I se presentan los valores de NISP, MNI y MNE identificados en cada una de las cuadrículas. Como puede observarse, las cuadrículas 2 y 3 son las que mayor densidad presentan y estos valores decrecen hacia el interior de la bahía. Asimismo, se pudo constatar una mayor cantidad de especímenes identificables en las cuadrículas I, 2 y 3 , y que el tamaño y volumen de los mismos decrece en las cuadrículas 4 y 5 , en las que aumenta la proporción de fragmentos indeterminados. 


\begin{tabular}{|c|c|c|c|c|c|c|c|c|}
\hline & \multicolumn{3}{|c|}{ Pinnipedia } & \multirow{2}{*}{$\frac{\text { L. guanicoe }}{\text { NISP }}$} & \multirow{2}{*}{$\begin{array}{l}\text { Ave } \\
\text { NISP }\end{array}$} & \multirow{2}{*}{$\frac{\text { B. taurus }}{\text { NISP }}$} & \multirow{2}{*}{ INDET } & \multirow{2}{*}{$\begin{array}{l}\text { NISP } \\
\text { Total }\end{array}$} \\
\hline & NISP & $\mathrm{MNI}$ & MNE & & & & & \\
\hline Cuadricula I & 336 & 10 & 234 & 2 & - & - & 28 & 366 \\
\hline Cuadricula 2 & 657 & 12 & 403 & 3 & 3 & - & 79 & 742 \\
\hline Cuadricula 3 & 440 & 8 & 245 & I & 7 & & 93 & 541 \\
\hline Cuadricula 4 & 230 & 4 & 79 & I & 22 & I & 72 & 326 \\
\hline Cuadricula 5 & 109 & 5 & 64 & I & 4 & - & 52 & 166 \\
\hline TOTAL & 1772 & 39 & 1025 & 8 & 36 & I & 324 & $214 \mid$ \\
\hline$\% N I S P$ & 82,76 & & & 0,37 & 1,68 & 0,04 & 15,13 & \\
\hline
\end{tabular}

Tabla 1: Composición taxonómica de las camas de hueso analizadas.

Table 1: Taxonomical composition of bone bed analyzed.

El MNI de lobos marinos en las 5 cuadrículas analizadas varía entre 12 y 4 por $\mathrm{m}^{2}$, totalizando 38 individuos, con un promedio de 7,6 por $\mathrm{m}^{2}$. Si tenemos en cuenta que las cuatro camas de huesos cubren unos $708 \mathrm{~m}^{2}$ y proyectamos los datos obtenidos hasta el momento, podemos estimar un MNI mínimo de 2194 , un $\mathrm{MNI}$ máximo de 8496 y un promedio de 5380 animales representados. La representación de las partes esqueletarias de los pinnípedos es bastante particular, con altos porcentajes de costillas y elementos de las extremidades anteriores (Tabla 2). Se pudo constatar la presencia de todas las categorías etarias, aunque la representación de animales muy pequeños parece ser considerablemente menor.

Se registró un patrón de meteorización con valores que oscilan entre el estadio 2 y 5 , siendo el estadio 4 el más frecuente en la muestra. Hay pérdida de tejido cortical en la mayor parte de los huesos, que presentan grietas, cuarteaduras y blanqueado.El avanzado estado de meteorización, sumado a que el análisis se llevó a cabo a la intemperie y en algunos momentos bajo la lluvia, no permite afirmar que no existan marcas de cortes o puntos de impacto, tal como fuera documentado en una factoría en isla Livingston, Antártida (Cartajena y Labarca 2007; Muñoz 2000). Las únicas alteraciones registradas que podrían interpretarse como de origen antrópico son unas perforaciones visibles en los cráneos, que probablemente sean producto de garrotazos para dar muerte a los animales. Si bien este atributo no fue cuantificado, podemos afirmar que la mayor parte de los cráneos lo presentaban (Figura 2d).

\section{CONSIDERACIONES FINALES}

Sin lugar a dudas, las particularidades de este sitio lo posicionan como un caso relevante para avanzar en la compresión sobre el impacto de la explotación industrial de pinnípedos en la región, para generar aportes a la discusión sobre tafonomía de pinnípedos o bien para realizar estudios isotópicos sobre un conjunto de características muy singulares, entre otros aspectos. Los datos presentado en este trabajo no agotan la informaciónobtenida en nuestra primera campaña y se prevé, en un próximo artículo en preparación, la incorporación de un cuerpo mayor de datos y su comparación con otros casos de estudios tafonómicos sobre pinnípedos (Borella y Muñoz 2006; Borella y Borrero 2010), con contextos históricos similares (Muñoz 2000; Cartajena y Labarca 2007), así como su discusión a la luz de marcos de referencia generados específicamente para pinnípedos (Borella et al 2008; San Román 2008). Sin embargo, debido a cuestiones de espacio hemos optado por una primera presentación sucinta y de carácter mayormente descriptivo a fin de dar a conocer las características más generales de este sitio.

Según las observaciones realizadas, parece defendible pensar que este conjunto es un depósito secundario, cuyo principal agente acumulador es la acciónde las mareas. El patrón distribucional de los restos - mayor densidad y tamaño de los especímenes en las cuadriculas externas- sumado a la configuración y morfología de la playa, sugiere la existencia de un ambiente de menor energía hacia el interior. Es probable que los trabajadores de la factoría descartasen los desechos del cocido de los 
animales hacia la bahía o en las playas adyacentes a los edificios y que la propia dinámica de las mareas haya depositado los huesos en la costa opuesta, concentrando los materiales más pesados en la zona de mayor energía y los ítems más livianos hacia la zona interior de la bahía.

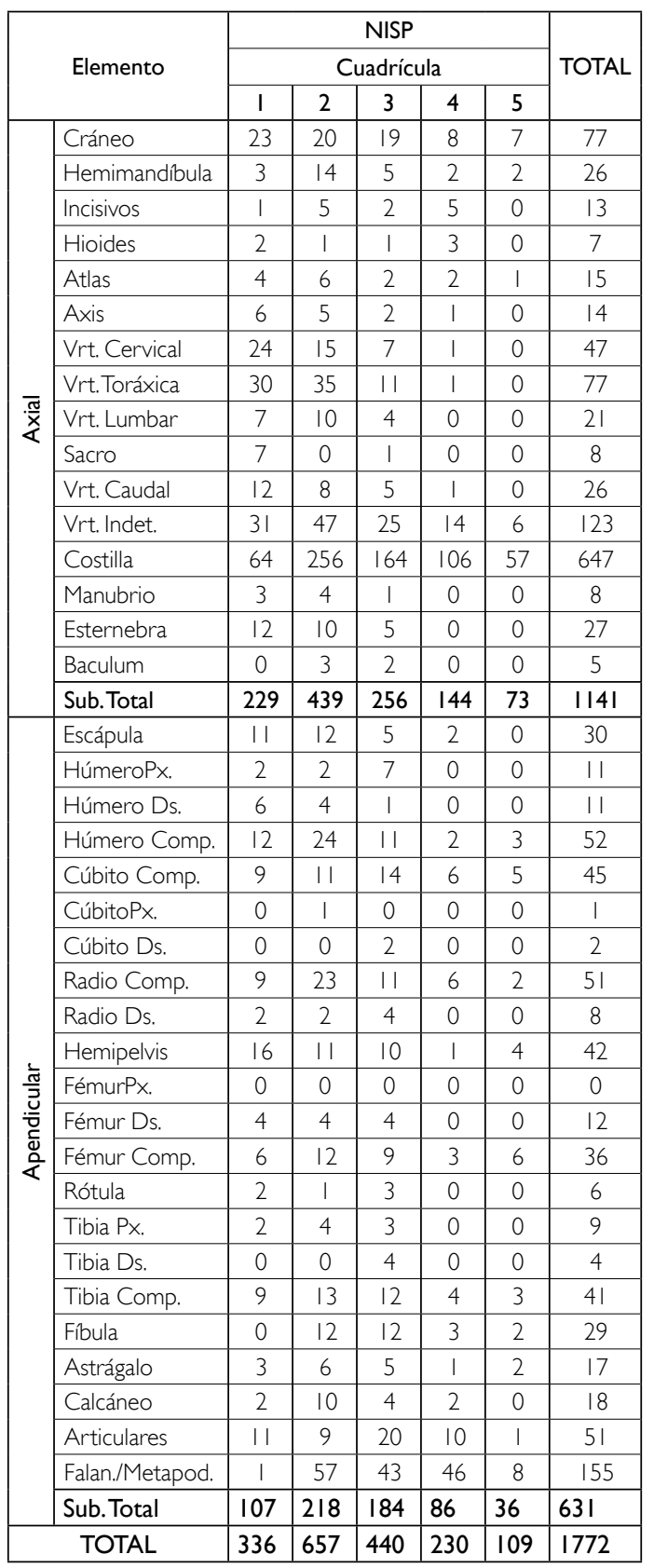

Tabla 2: Composición anatómicade pinnípedos en las cuadriculas analizadas.

Table 2:Anatomical composition of pinnipeds in the grids analyzed.
Los avanzados estadios de meteorización registrados, así como la baja representación de algunos elementos, nos hace suponer que este conjunto ha sido afectado por intensos procesos atricionales, los cuales estarían afectando la representación anatómica y etaria del conjunto.

Consideramos que este sitio tiene un alto potencial para realizar estudios zooarqueológicos y tafonómicos. Si bien las distancias y las dificultades logísticas para sostener campañas regulares en la región son grandes, las posibilidades de monitorear procesos de enterramiento, avance de los estadios de meteorización, presencia de indicadores de estabilidad, pérdida, agregados y desplazamiento de materiales, entre muchos otros objetivos que podrían integrar una agenda de trabajo. En este sentido -además del potencial de reconstrucción sobre un registro histórico poco conocido- la continuidad de los trabajos en este sitio persigue el objetivo de generar nuevos aportes a la discusión sobre la tafonomía de pinnípedos.

\section{AGRADECIMIENTOS:}

A Apache Energía Argentina S.R.L. y Total Austral S.A. por el financiamiento. Fundación Génesis, Secretaría de Cultura de Tierra del Fuego, Museo del Fin del Mundo. Adolfo Imbert (Centro Hípico Ushuaia). A dos evaluadores anónimos que con sus comentarios enriquecieron y mejoraron el manuscrito original.

\section{Notas}

'Si bien estas colonias han sido definidas como de Otaria flavescens (Carrara 1952), A. Winograd (1984) no descarta la presencia de Arctocephalus australis en la zona en tiempos previos a su explotación industrial. Por otra parte, las condiciones en las que se realizaron los trabajos de campo no permitieron determinar con seguridad la presencia de especímenes correspondientes a A. australis, sin embargo sobre la base de criterios de tamaño, consideramos que una proporción de los especímenes analizados podría pertenecer a esta especie, razón por la cual hemos preferido realizar nuestro análisis utilizando la categoría más inclusiva de pinnípedos. 


\section{BIBLIOGRAFÍA}

Borella, F. y L. A. Borrero 2010. "Observaciones tafonómicas acerca de la desarticulación de carcasas de pinnípedos en ambientes litorales, el caso del islote Lobos (Golfo San Matías, Rio negro)". En:Zooarqueología a Principios del Siglo XXI:Aportes Teóricos, Metodológicos y Casos de Estudio, editado por M. A. Gutiérrez, M. De Nigris, P. Fernández, M. Giardina, A. Gil, A. Izeta, G. Neme and H. Yacobaccio. PP 37I-379. Ediciones del Espinillo,Buenos Aires.

Borella, F.; M. A. Gutiérrez; H. R. Fodere y J. F. Merlo 2008. "Estudios de densidad mineral ósea para dos especies de otáridos frecuentes en el registro arqueofaunístico patagónico". En: Arqueología de Fuego-Patagonia. Levantando piedras, levantando huesos... y develando arcanos, editado por F. Morello, A. Martinic, A. Prieto y G. Bahamonde. PP. 421-426. ediciones CEQUA, Punta Arenas.

Borella, F y A. S. Muñoz 2006. "Observaciones tafonómicas sobre restos de pinnípedos en la costa norte fueguina (Argentina)". Intersecciones en Antropología 7:399-403.

Bruno Videla, P.1980."Recursos naturales de Tierra del Fuego: Lobos marinos". Colaboración al $3^{\circ}$ Congreso de Ciencias Históricas Fueguinas. Ushuaia. Manuscrito en posesión de los autores.

Carrara, I. S.1952.Lobos marinos, pingüinos y guaneras de las costas del litoral marítimo e islas adyacentes de la República Argentina. Universidad Nacional de La Plata. Facultad de Ciencias Veterinarias. Cátedra de Higiene e Industria (Publicación especial). La Plata.

Cartajena, I. y R. Labarca. 2007."Patrones históricos de cuereo en lobos marinos: la evidencia de los abrigos I y 2 de Playa Yámana (isla Livingston, antártica chilena)”. Magallania35 (I):95-105.

Fisher, J.W. 1995. "Bone surface modifications in zooarchaeology".Journal of Archaeological Method and Theory.2, (I):7-68.
Lyman, R.L. 1994. Vertebrate Taphonomy.Cambridge University Press, Cambridge.

MengoniGoñalons, L. 1999. Cazadores de Guanacos de la Estepa Patagónica. Sociedad Argentina de Antropología. Sociedad Argentina de Antropología. Buenos Aires.

Muñoz, A. S. 2000."Zooarqueología de la isla Livingston. Shetland del Sur.'Archaeofauna 9: 39-57.

San Román, M. 2008. "Anatomía económica de Otaria flavescens (Shaw, I800)". En: Zooarqueología y Tafonomía en el Confin del Mundo, editado por M. López, I. Cartajena, C. P. García y F. Mena. Pp. 169-179. Monografías Arqueológicas $N^{\circ}$ I, facultad de estudios del Patrimonio Cultural, Universidad Internacional SEK, Santiago de Chile.

Stehberg, R. y A. Cabeza. 1987. "Comienzos de la arqueología histórica antártica en el sitio Cuatro Pircas". Revista Chilena de Antropología. 6, 83-III.

Vázquez, M., M. Álvarez, R. Barberena, K. Borrazzo, L. A. Borrero, D. Elkin, M. Grosso, C. Murray, J. Oría, M.

Salemme y F. Santiago.2010. "Programa arqueológico costa atlántica: hacia la preservación del patrimonio arqueológico costero en Tierra del Fuego". En: Arqueología en el Bicentenario de la Revolución de Mayo, editado por R. Bárcena y H. Chiavazza, Tomo II, Pp. 557-562. XVII Congreso Nacional de Arqueología Argentina. Facultad de Filosofía y Letras, Universidad Nacional de Cuyo, Mendoza.

Winograd, A. J. 1984."Presencia de pinnípedos en el litoral septentrional”. En: PEOAF 1984. Informe Museo Territorial. Pp. 70-I0I. Ushuaia.

Winograd, A, J. 2008. Patagonia. Mitos y Certezas. Edhasa. Buenos Aires.

Zarankin, A. y Senatore, X. 2007. Historias de un Pasado en Blanco:Arqueología Histórica Antártica. Argvmentvm, Belo Horizonte. 